\title{
THz Time Domain Spectroscopy of Thin Gold Layers on GaAs
}

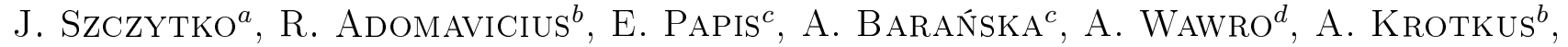 \\ B. PiEcTKA ${ }^{a}$ AND J. ŁUSAKOWSKI ${ }^{a}$ \\ ${ }^{a}$ Institute of Experimental Physics, Faculty of Physics, University of Warsaw, Hoża 69, 00-681 Warsaw, Poland \\ ${ }^{b}$ Center for Physical Sciences and Technology, A. Gostauto 11, 01108, Vilnius, Lithuania \\ ${ }^{c}$ Institute of Electron Technology, al. Lotników 32/46, 02-668 Warsaw, Poland \\ ${ }^{d}$ Institute of Physics, PAS, al. Lotników 32/46, 02-668 Warsaw, Poland
}

\begin{abstract}
Thin layers of $\mathrm{Au}$ with the thickness of several nanometers were prepared on a semi-insulating GaAs substrate. The layers' thickness was determined by ellipsometry. $\mathrm{THz}$ time-domain spectroscopy was applied to determine a complex index of refraction of thin Au layers. The obtained results allow for a more precise modeling of the performance of semiconductor devices at $\mathrm{THz}$ frequencies.
\end{abstract}

PACS: 78.66.Bz, 78.68. $+\mathrm{m}$

\section{Introduction}

Semiconductor micro- and nanostructures are very promising candidates for detectors and emitters in $\mathrm{THz}$ range of the electromagnetic spectrum [1]. The basic underlying mechanism is a coupling of electromagnetic waves to oscillations of the electron plasma in the semiconductor. As an example, it was proved that field-effect transistors (FETs) can be used as THz detectors at room as well as cryogenic temperatures. Such a coupling of photons to plasmons is usually achieved with a planar metallic antenna connected to the source and gate. In this context, a question arises about $\mathrm{THz}$ properties of thin metallic layers deposited on a semiconductor surface which form antenna pads.

Numerical modeling appears to be an indispensable tool to understand $\mathrm{THz}$ properties of a FET. THz properties of materials used to fabricate a FET are the input data for computer simulations. On the micro- and submicrometer level, properties of a semiconductor structure or a metallic layer depend on the technology. For this reason, one should determine properties of layers obtained within a technology that will subsequently be used to produce a FET.

We addressed the problem of $\mathrm{THz}$ properties of thin $\mathrm{Au}$ layers deposited on a GaAs. Such layers form gates semitransparent at $\mathrm{THz}$ frequency and antenna metallization pads. The final goal of the investigation is to determine a complex index of refraction of $\mathrm{Au}$ deposited on a GaAs substrate in the $\mathrm{THz}$ range. The experimental tool was a time-domain spectroscopy based on a fs-laser pulsed system.

\section{Experiment}

A series of Au layers was prepared on a (100) surface of an epiready semi-insulating GaAs of $320 \mu \mathrm{m}$ thickness by a dc magnetron sputtering using a Leybold L400cp system. The deposition process was performed in $\mathrm{Ar}^{+}$ plasma at working pressure $3 \times 10^{-3}$ mbar, a gas flow of $\mathrm{Ar}^{+}=100 \mathrm{sccm}$ and the power of $300 \mathrm{~W}$. The thickness of the Au layers was measured by ellipsometry and was equal to $8.7 \mathrm{~nm}$ and $18.6 \mathrm{~nm}$. Such a small thickness was used to enable transmission experiments in the $\mathrm{THz}$ range. We have verified that the layers thicker than $50 \mathrm{~nm}$ are opaque to $\mathrm{THz}$ radiation.

The experiment was performed with so-called $\mathrm{THz}$ time domain spectroscopy method ( $\mathrm{THz}$ TDS). A photoconductive $\mathrm{THz}$ emitter excited by a $150 \mathrm{fs}$ duration, $76 \mathrm{MHz}$ repetition rate, and $810 \mathrm{~nm}$ central wavelength pulses from a mode-locked Ti:sapphire laser was used as a $\mathrm{THz}$ source in the range $0.1-1.5 \mathrm{THz}$. THz radiation was transmitted through the sample placed in an optical cryostat and collected by an ultrafast photodetector with a dipole antenna and a substrate lens made from a high resistivity silicon. The detector was manufactured from a low-temperature GaAs grown by the molecular beam epitaxy at about $250^{\circ} \mathrm{C}$ substrate temperature. It showed an electron trapping time of about $200 \mathrm{fs}$, thus the signal at the photodetector appears when the carriers are present shortly after the excitation by fs pulse. The fs photoexcited carriers are driven by $\mathrm{THz}$ electric field interacting with dipole antenna, they reach electric contacts and finally are detected by lock-in amplifier.

\section{Results}

In a THz TDS experiment, one records a voltage on the photodetector as a function of a delay between the $\mathrm{THz}$ pulse and the reference ultrashort fs (duration less than $40 \mathrm{fs}$ ) pulse from a Ti:sapphire laser. The spectrum consists of several peaks (with positive or negative sign) with the amplitude decreasing with the increase of the delay time, which originate from multiple reflections of $\mathrm{THz}$ radiation inside the sample (Fig. 1). The time interval between successive peaks, as well as their phase depends on the thickness of the sample and its complex index of refraction. The strongest signal is obtained when the light 
transmitted through the sample without any internal reflection arrives at the photodetector at the same time as a reference $\mathrm{fs}$ pulse. Positive and negative sign of voltage means that the phase of $\mathrm{THz}$ electric field recorded by the dipole antenna varies with delay time.

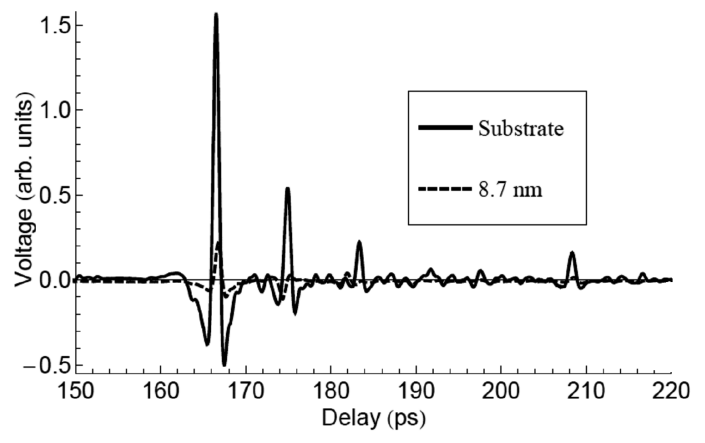

Fig. 1. Results of a THz TDS experiment on GaAs sample. Voltage signal of transmission through the substrate is compared with voltage signal of transmission through Au layers of $8.7 \mathrm{~nm}$ thick.

\section{Data analysis}

In order to obtain a spectrum in the energy domain, rather than in the time domain, one has to Fourier transform time-dependent results (Fig. 2). In our case, we have got spectra dominated by a Fabry-Perot etalon pattern resulting from interferences in the GaAs substrate. The spectrum consists of several peaks with the amplitude decreasing with the increase of the frequency (Fig. 2). A reference spectrum was obtained by measurements of the transmission without the sample. The signal processed by a numerical analysis was a spectrum of transmission through a sample ("sample spectrum") divided by the reference spectrum.

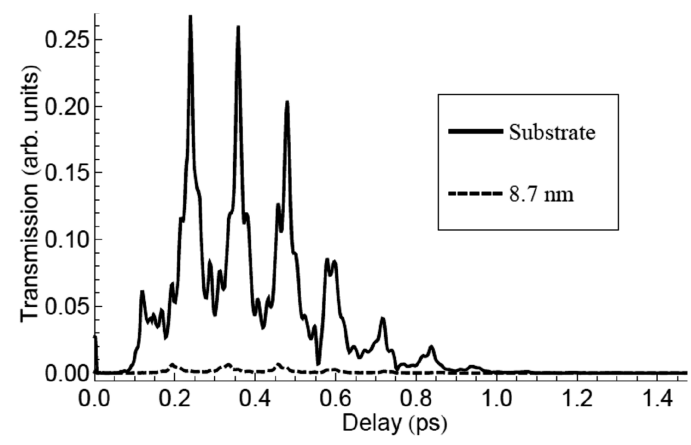

Fig. 2. Results of a Fourier transformation of a time-domain spectrum. The transmission from the substrate is compared with the transmission of Au layer of $8.7 \mathrm{~nm}$.

The transmission data were analyzed with a standard transfer matrix method of the form of

$$
M_{m}=\frac{1}{t_{m-1, m}}\left[\begin{array}{cc}
1 & r_{m-1, m} \\
r_{m-1, m} & 1
\end{array}\right]\left[\begin{array}{cc}
\mathrm{e}^{-\mathrm{i} \delta_{m}} & 0 \\
0 & \mathrm{e}^{\mathrm{i} \delta_{m}}
\end{array}\right],
$$

where $M_{m}$ is a transfer matrix through the layer $m$ by light passing from layer denoted as $m-1$; the symbols $t_{m-1, m}$ and $r_{m-1, m}$ represent, respectively, amplitude transmission and reflection coefficients between the neighboring layers. Since we used a configuration of a normal incidence, TM and TE transmission and reflection coefficients are the same. The term $\mathrm{e}^{-\mathrm{i} \delta_{m}}$ represents propagation of the light in the medium $m$. Relative amplitudes of the transmitted and reflected waves at an interface between two media are given by Fresnel's coefficients depending on refractive indexes $n_{m-1}$ and $n_{m}$. The term $\delta_{m}=n_{m} d_{m} \frac{\omega}{c}$ represents an optical path length, where $c$ is the light velocity, $\omega$ is the frequency and $d_{m}$ is the thickness of the layer.

In the case of dissipative media - like metallic layers - refractive indexes are complex. By modeling the dependence of $n_{m}$ on the frequency $\omega$ one can obtain the transmission coefficient $T(\omega)$ of the measured multilayered system.

For a GaAs substrate we took a real value of $n_{\mathrm{GaAs}}=$ 3.6. For an $\mathrm{Au}$ layer we took a dependence given by the Drude model of dielectric function $\varepsilon(\omega)$ of damped plasma frequency [2-4]:

$$
n^{2}(\omega)=\varepsilon(\omega)=1-\frac{\omega_{\mathrm{p}}^{2}}{\omega^{2}+\mathrm{i} \omega \omega_{\tau}},
$$

where $\omega_{\mathrm{p}}$ is the plasma frequency of gold and $\omega_{\tau}$ is its damping frequency. It is not possible to obtain $\omega_{\mathrm{p}}$ and $\omega_{\tau}$ independently since both are related to the so-called optical conductivity $\sigma_{\mathrm{opt}}=\varepsilon_{0} \frac{\omega_{\mathrm{p}}^{2}}{\omega_{\tau}}$. Therefore we assumed bulk value of $\omega_{\mathrm{p}}=2186 \mathrm{THz}$, which was used for photons energies $10-660 \mathrm{THz}[3-5]$ and we fitted only $\sigma_{\mathrm{opt}}$.

The measured intensity $I_{\mathrm{GaAs} / \mathrm{Au}}^{\exp }(\omega)$ of the $\mathrm{GaAs} / \mathrm{Au}$ spectrum in Fig. 2 consists of the transmission coefficient $T_{\mathrm{GaAs} / \mathrm{Au}}(\omega)$ and unknown envelope of the intensity of the THz source $I_{0}^{\exp }(\omega)$ :

$$
I_{\mathrm{GaAs} / \mathrm{Au}}^{\exp }(\omega)=T_{\mathrm{GaAs} / \mathrm{Au}}(\omega) I_{0}^{\exp }(\omega) \text {. }
$$

In order to obtain $I_{0}^{\exp }(\omega)$ we also measured the intensity of the $\mathrm{THz}$ radiation through the GaAs substrate $I_{\mathrm{GaAs}}^{\exp }(\omega)$ and modeled transmission $T_{\mathrm{GaAs}}(\omega)$. Finally, we fitted parameters of gold in the spectrum $I_{\mathrm{GaAs} / \mathrm{Au}}^{\exp }(\omega)$ divided by $I_{\mathrm{GaAs}}^{\exp }(\omega)$ :

$$
\frac{I_{\mathrm{GaAs} / \mathrm{Au}}^{\exp }(\omega)}{I_{\mathrm{GaAs}}^{\exp }(\omega)}=\frac{T_{\mathrm{GaAs} / \mathrm{Au}}(\omega)}{T_{\mathrm{GaAs}}(\omega)} \text {. }
$$

We found that it was difficult to obtain good results using the spectra $I_{\mathrm{GaAs}}^{\exp }(\omega)$ measured on the same substrate thickness as in $\mathrm{GaAs} / \mathrm{Au}$ sample. The reason is that $T_{\mathrm{GaAs}}(\omega)$ is a transmission of a Fabry-Perot etalon - which is just periodic function of optical path length $\delta_{\text {GaAs }}$ and thus of $\omega$. Therefore, if periods of $T_{\mathrm{GaAs} / \mathrm{Au}}(\omega)$ and $T_{\mathrm{GaAs}}(\omega)$ are too close one to the other, then an experimental uncertainty of the measured values $I_{\mathrm{GaAs} / \mathrm{Au}}^{\exp }(\omega)$ and $I_{\mathrm{GaAs}}^{\exp }(\omega)$ strongly modifies quotient results. Such uncertainty can be for instant created by a small difference between the angle of incidence of the $\mathrm{THz}$ radiation passing through $\mathrm{GaAs} / \mathrm{Au}$ and $\mathrm{GaAs}$ and 
therefore slightly different optical path length in GaAs substrate. Intentionally, the light was passing through the sample at the normal incidence, however it was not controlled with a precision better than 10 degrees.

The best solution was to measure $I_{\mathrm{GaAs}}^{\exp }(\omega)$ on a substrate of an essentially different thickness $d_{\mathrm{GaAs}}=$ $350 \mu \mathrm{m}$ than the thickness of the $\mathrm{GaAs} / \mathrm{Au}$ sample $(320 \mu \mathrm{m})$. Thus small deviation from normal angle of incidence of $\mathrm{THz}$ radiation was corrected in calculations by the small deviation of the nominal thickness of GaAs substrate (below $2 \%$ ), which - contrary to the situation described before - did not change significantly the shape of the $I_{\mathrm{GaAs} / \mathrm{Au}}^{\exp }(\omega)$ and $I_{\mathrm{GaAs}}^{\exp }(\omega)$ ratio.

The results of the experimental ratio $I_{\mathrm{GaAs} / \mathrm{Au}}^{\exp }(\omega) /$ $I_{\mathrm{GaAs}}^{\exp }(\omega)$ and calculated $T_{\mathrm{GaAs} / \mathrm{Au}}(\omega) / T_{\mathrm{GaAs}}(\omega)$ are presented in Fig. 3.

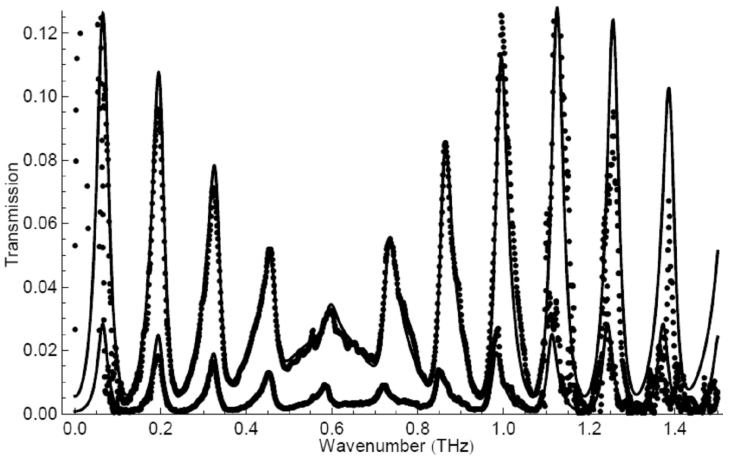

Fig. 3. The results of the fit (solid lines) of Eq. (1) to the experimental data - upper points: spectrum $I_{\mathrm{GaAs} / \mathrm{Au}}^{\exp }(\omega) / I_{\mathrm{GaAs}}^{\exp }(\omega)$ of $\mathrm{GaAs} / \mathrm{Au} 8.7 \mathrm{~nm}$, lower points - spectrum of GaAs/Au $18.6 \mathrm{~nm}$.

We varied the thickness of GaAs substrate $d_{\mathrm{GaAs}}$ (nominally $320 \mu \mathrm{m}$ ), and the refractive index of $\mathrm{Au}$ layer $\left(n_{\mathrm{Au}}\right)$. We assumed values of $d_{\mathrm{Au}}$ taken from the ellipsometry experiments. Fitting the amplitude of the etalon spectrum and the distance between maxima one can get the full information about the thickness of the sample and the complex indexes of refraction of constituent layers. The obtained parameters for $\mathrm{Au}$ are presented in Table (frequency $\nu$ is given in $\mathrm{THz}, \omega=2 \pi \nu$ ).
Summary of the fitting procedure.

TABLE

\begin{tabular}{c|c|c|c|c}
\hline \hline $\begin{array}{c}d_{\mathrm{Au}}[\mathrm{nm}] \\
(\text { ellipsometry) }\end{array}$ & $\begin{array}{c}\nu_{\mathrm{p}} \mathrm{Au} \\
{[\mathrm{THz}]}\end{array}$ & $\begin{array}{c}\nu_{\tau} \mathrm{Au} \\
{[\mathrm{THz}]}\end{array}$ & $\begin{array}{c}\sigma_{\mathrm{opt}} \\
{\left[(\Omega \mathrm{cm})^{-1}\right]}\end{array}$ & $n_{\mathrm{Au}}[1 \mathrm{THz}]$ \\
\hline 18.6 & 2186 & 34.5 & 770000 & $259.3+266.9 \mathrm{i}$ \\
8.7 & 2186 & 38.2 & 700000 & $246.8+253.3 \mathrm{i}$
\end{tabular}

The bulk value of the optical conductivity for the wave number range of $10-10^{5} \mathrm{~cm}^{-1}$ is about 411000 $450000(\Omega \mathrm{cm})^{-1}([4]$ and references therein $)$.

\section{Conclusions}

The results of transmission of $\mathrm{THz}$ radiation over $\mathrm{Au}$ layers were analyzed with a transfer matrix method which allowed for determination of the refractive index of $\mathrm{Au}$. From these investigations the optical parameters of $\mathrm{Au}$ thin layers were determined. The results allow for a better modeling of devices and understanding their $\mathrm{THz}$ response.

\section{Acknowledgments}

This work was partially supported by a Foundation for Polish Science grant POMOST and a Polish Ministry of Science and Higher Education grant N N515 608139.

\section{References}

[1] W. Knap, M. Dyakonov, D. Coquillat, F. Teppe, N. Dyakonova, J. Lusakowski, K. Karpierz, M. Sakowicz, G. Valusis, D. Seliuta, I. Kasalynas, A. El Fatimy, Y. M. Meziani, T. Otsuji, J. Infrared Milli Terahertz Waves 30, 1319 (2009).

[2] P.B. Johnson, R.W. Christy, Phys. Rev. B 6, 4370 (1972).

[3] M.A. Ordal, L.L. Long, R.J. Bell, S.E. Bell, R.R. Bell, R.W. Alexander, Jr., C.A. Ward, Appl. Opt. 22, 1099 (1983).

[4] M.A. Ordal, R.J. Bell, R.W. Alexander, Jr, L.L. Long, M.R. Querry, Appl. Opt. 24, 4493 (1985).

[5] Landolt-Bornstein Database, "Electronic structure and transport - 4. Optical properties of pure metals and binary alloys - 4.2 Drude parameters of pure metals", Springer Materials Database, p. 214. 